\title{
Vibrotactile threshold measurement for detecting peripheral neuropathy: defining variability and a normal range for clinical and research use
}

\author{
J. Duke • M. McEvoy • D. Sibbritt • M. Guest • \\ W. Smith • J. Attia
}

Received: 22 May 2007 / Accepted: 23 July 2007 / Published online: 11 September 2007

(C) Springer-Verlag 2007

\begin{abstract}
Aims/hypothesis We aimed to define normal ranges for vibration sense as measured by vibratory perception thresholds (VPTs) using biothesiometry.

Methods We performed biothesiometry in a communitydwelling sample of 901 people aged 55 to 85 years who did not have diabetes. We quantitated the variation between repeat measures using analysis of variance and BlandAltman plots. We also plotted the age- and sex-specific reference ranges.

Results We found small but statistically significant differences between repeat measures using the ascending and descending method of limits. Statistically higher vibration thresholds were noted on the right arm and leg compared with the left. Significantly higher vibration thresholds were also seen in men vs women for both lower limbs. We also defined sex-specific reference intervals (normal ranges) for biothesiometry for older persons and quantitated the increase in vibration threshold with increasing age.

Conclusions/interpretation For reliability, it may be sufficient to obtain the average of two ascending measures separated by at least $1 \mathrm{~min}$ in just the right hand or right foot, since this is usually the one with the higher threshold. Although identical reference ranges can be used for men and women for the upper limb, there are significant differences in the lower limbs. The major determinant of
\end{abstract}

J. Duke $\cdot$ M. McEvoy $\cdot$ D. Sibbritt $\cdot$ M. Guest $\cdot$ W. Smith $\cdot$ J. Attia Centre for Clinical Epidemiology and Biostatistics,

David Maddison Building, University of Newcastle,

Newcastle, New South Wales, Australia

\section{J. Attia $(\square)$}

Division of Medicine, John Hunter Hospital,

Locked Bag 1, Hunter Region Mail Centre,

New Lambton Heights 2310 New South Wales, Australia

e-mail: john.attia@newcastle.edu.au
VPT is age: we have established age-specific norms for VPT testing for adults between 55 and 85 years of age.

Keywords Biothesiometry · Neuropathy · Normal range ·

Peripheral nerve $\cdot$ Reference interval .

Quantitative sensory testing - Vibration sense .

Vibratory perception threshold - Vibrotactile threshold . VPT

\author{
Abbreviations \\ HCS Hunter Community Study \\ VPT vibratory perception threshold
}

\section{Introduction}

Peripheral neuropathy occurs as a complication of diabetes, a component of several common and many rare diseases, and also as an adverse health outcome from exposure to various chemical substances or physical factors in the workplace [1-3]. The population prevalence of peripheral neuropathy has been reported to be approximately $2.4 \%$, rising with age to $8 \%$ [4]. Most peripheral neuropathies are chronic and usually develop over several months [5].

Peripheral neuropathy is one of the least well-understood complications of diabetes, with an estimated prevalence of $13.1 \%$ in patients with known diabetes and $7.1 \%$ in newly diagnosed diabetic patients [6]. The most common form of peripheral neuropathy in diabetes is the distal symmetric polyneuropathy often described as the stocking-glove neuropathy, affecting first the longest nerves and then progressing proximally [7]. While sensory, motor and autonomic functions may be affected, it is the loss of sensory signals that poses the greatest threat to the limb [7]. Risk factors associated with neuropathy include poor 
glycaemic control, insulin use, duration of diabetes, age, alcoholism and height $[6,8]$.

Various methods have been used to assess peripheral neuropathy including physical assessment, quantitative vibration perception testing and electrophysiological evaluation. Physical assessment can be performed quickly but is non-quantitative, of unknown reliability and requires a highly skilled assessor. Electrophysical methods, which are used to test the largest myelinated sensory and motor fibres, are quantitative and reliable, but require sophisticated equipment, skilled technicians and are time-consuming [9, 10]. Quantitative vibration perception testing, on the other hand, can be done relatively quickly using inexpensive equipment by an assessor with minimal training [10, 11]. Vibration is sensed by activation of the Meissner and Pacinian corpuscles, these signals being also conducted by the same large myelinated sensory fibres tested by electrophysical methods. The time taken to conduct vibratory perception threshold (VPT) measurements is considered to be relatively fast. However, the uptake of VPT for clinical use and in research studies has been affected by various factors. These include lack of understanding of the variability in VPT measurements, clinical and demographic factors affecting VPT, and the lack of clear reference ranges for the variety of instruments used in VPT. It is worth noting that neither electrophysical nor VPT methods measure the slow-conducting, unmyelinated or thinly myelinated fibres that conduct thermal sensation. These can be tested using quantitative sensory testing for temperature.

In this study, VPTs were obtained at multiple sites across upper and lower limbs and on the right and left sides. Our aims were: (1) to assess the intra-individual variability of VPTs within each site and across sites, comparing right and left sides for bilateral upper and lower limb sites tested; (2) to compare variability between upper and lower limbs; and (3) to assess whether variability in VPT changes with sex and advancing age.

\section{Methods}

Participants and study protocol Data for this study come from Phase I of the Hunter Community Study (HCS), a cohort of community-dwelling men and women aged 55 to 85 years in Newcastle, NSW, Australia. Participants were randomly selected from the NSW State Electoral roll and contacted between mid-December 2004 and June 2005. A modified Dillman [12] recruiting strategy was used, whereby two letters of introduction and an invitation to participate were posted to the selected persons. Persons who did not respond to initial postal contacts were telephoned by an HCS research assistant. If contact was not established after five attempts, the individual was classified as a non-responder. Persons who could not speak English were deemed ineligible.

Once consent to participate was obtained from eligible study participants, they were asked to complete a series of self-report postal questionnaires containing details on demographics, morbidity, complementary and alternative medicines and medication use, health professional utilisation, a food-frequency questionnaire, as well as the following: Short Form 36 [13], Physical Activity Scale for the Elderly [14, 15], Kessler Psychological Distress scale (K 10) [16, 17], Centre for Epidemiological Studies Depression scale [18, 19], Memory Complaint Questionnaire [20], Duke Social Support Index [21, 22] and Australian Quality of Life-Mark 2 [23].

Participants were then invited to attend the HCS data collection centre to enable a series of clinical measurements to be ascertained. Consent to linkage to data from the Health Insurance Commission (Medicare and Pharmaceutical Benefit Scheme) and local health databases was also sought at this time. The clinical measurements assessed were: blood pressure, height, weight, waist circumference, peripheral vibration sensory testing, mini-mental status examination, spirometry, olfaction, hearing, vision, functional reach, grip strength, mobility and routine blood values (including cholesterol and fasting blood glucose level, but not $\mathrm{HbA}_{1 \mathrm{c}}$ ). Participants were also asked to wear a pedometer and keep a 7 day diary of step counts.

Of 2253 potential participants approached after random selection from the electoral roll, $1071(48 \%)$ agreed to participate, for 901 of whom (40\%) data were collected and completed. Comparisons between responders and nonresponders demonstrated no statistical significant difference for sex (men $46.1 \%$ vs women $46.9 \%$ ). However, a difference was demonstrated for age, in that responders were slightly younger than non-responders (mean 66.3 [SD 7.8] years vs 68.6 [SD 8.4] years, $p<0.001$ ). The HCS participants provide a population profile approximately reflecting that of the Hunter (NSW, Australia) [24], state [24] and national [25] Australian profiles. Specifically, there were no statistically significant differences between the HCS and the Hunter, state and national profiles in terms of sex distribution, but the HCS participants were slightly younger than the Hunter $(p<0.001)$, state $(p=0.02)$ and national $(p=0.002)$ profiles.

This research was conducted as part of the HCS, which has been approved by the University of Newcastle Human Research Ethics Committees.

Measurement of peripheral neuropathy The biothesiometer (50-60 Hz; Model PVD-LP; Bio-Medical Instrument Company, Newbury, OH, USA) is a clinically validated method of rapid screening, early detection and longitudinal 
evaluation of persons at risk of sensory dysfunction associated with disease and occupational injury, and has been used in other studies of diabetes and peripheral neuropathy [26]. It is a rheostat attached to a vibrating probe; as the voltage is increased, the amplitude of the vibration also increases.

Participants were asked to remove their shoes, socks or stockings and lie in a supine position with knees bent so that their feet were flat on the bed. The probe was held at $90^{\circ}$ to the skin with constant pressure. Three sites of each lower limb were used: hallux distal interphalangeal (big toe), medial malleolus (ankle) and medial tibial plateau (knee). One trial run with the biothesiometer was initially conducted at a site other than those mentioned above, most commonly the shoulder, in order to demonstrate to the participant the expected sensation. The testing protocols began on the right leg and right arm, respectively. If a VPT of $\leq 50 \mathrm{~V}$ was recorded at the first site (e.g. hallux distal interphalangeal), no further lower limb sites were tested.

The method of limits was applied, whereby the voltage was slowly increased from $0 \mathrm{~V}$ at a rate of $1 \mathrm{~V} / \mathrm{s}$ to each limb consecutively. VPT was the recorded voltage at the moment the participant indicated that he or she first felt the vibration (first ascending reading). The voltage was then increased to 10 $\mathrm{V}$ above the first recorded VPT and then slowly reduced till the vibration was no longer felt (second descending recording) on first limb. The method was then applied to record the first two measurements on the other limb. The final VPT measurement (third ascending reading) was then recorded on the first limb by ascending voltage. The testing was then completed on the other limb. The method of limits was then applied to upper limbs with joints of the middle finger, distal interphalangeal (fingertip), metacarpophalangeal (knuckle), radial styloid (wrist) and olecranon (elbow). The hand was rested on a rice-filled pillow. If a VPT of $\leq 50 \mathrm{~V}$ was felt at the first joint (e.g. distal interphalangeal), no further upper limb sites were tested. The participant was supine throughout the testing procedure and could not see the biothesiometer controls. Testing was done in an air-conditioned room at 22 to $24^{\circ} \mathrm{C}$.

Statistical analyses Repeated measures analysis of variance was used to test for differences between repeated VPT measurements (two ascending and one descending). If this was positive, associated pairwise comparisons between ascending and descending distal measurements were made using paired $t$ tests, with adjustment for multiple comparisons. Paired $t$ tests were also used to test for differences between the left and right distal VPT measurements. Linear regression lines, with $95 \%$ CIs, were used to demonstrate the relationship between the distal measurements and age. Pearson's correlation coefficient was used to measure the association between right and left limb measurements.
Bland-Altman plots were used to assess variability, with the average of the three readings on the $x$-axis and the difference between two measurements of interest on the $y$-axis. All statistical analyses were conducted using Stata version 9 (StataCorp LP, College Station, TX, USA).

\section{Results}

Participant characteristics Phase I of the HCS contained information on 901 participants. Participants with selfreport of diabetes or taking diabetic medications $(n=105)$, stroke $(n=35)$ and/or heart disease $(n=112)$ (not mutually exclusive groups) were excluded, leaving 662 participants. The average age of the remaining participants was 66.1 $(\mathrm{SD}=7.6)$ years and $56.0 \%$ were women.

Lower limb results Table 1 shows the mean (SD) of the distal leg measurements. There were 589 participants with a distal leg measurement. These findings are reported separately for the left and right limbs, by age group. For both limbs separately, there were statistically significant differences in the total means between: (1) the first ascending measurement and the descending measurement $(p<0.001)$; (2) the first ascending measurement and the second ascending measurement $(p<0.001)$; and (3) the descending measurement and the second ascending measurement $(p<0.001)$. For both limbs, the mean of the first ascending measurement was the highest value, followed by the second ascending measurement, then the descending measurement. A Bland-Altman plot (Fig. 1) confirmed the significant but clinically small difference between the first ascending and descending readings in the lower limb; this difference remained constant throughout the entire range of values, as did the spread of values.

In comparing the total means between the left and right limbs, there were statistically significant differences between the first ascending measurements $(p=0.04)$ and the descending measurements $(p=0.01)$, but no statistically significant difference between the first and second ascending measurements $(p=0.14)$. In all three comparisons between the left and right limbs, the right limb had the highest mean values. Note that only six participants had ankle and knee measurements; these were not analysed.

Figure 2 shows the relationship between the distal leg measurements and age, separately for both sexes. There was a statistically significant linear relationship between distal leg measurements and age, for both men $(p<0.001)$ and women $(p<0.001)$, with voltage increasing with age. As seen from the linear regression lines and their corresponding 95\% CIs, there was a statistically significant difference in voltage between men and women from the age 
Table 1 Distal measurements summary for left and right leg, stratified by participants aged $\leq 70$ years and those aged $>70$ years

\begin{tabular}{|c|c|c|c|}
\hline Distal measurement & $\leq 70$ years & $>70$ years & Total \\
\hline \multicolumn{4}{|l|}{ Right leg } \\
\hline Ascending 1 & $16.1(7.6)$ & $21.9(10.9)$ & $17.8(9.3)$ \\
\hline Descending 1 & $15.0(7.6)$ & $20.2(11.0)$ & $16.5(9.3)$ \\
\hline Ascending 2 & $15.2(7.5)$ & $20.9(10.9)$ & $16.8(9.2)$ \\
\hline Average, all three & $15.4(7.4)$ & $21.0(10.6)$ & $17.0(9.1)$ \\
\hline Average, ascending & $15.6(7.4)$ & $21.4(10.7)$ & $17.3(9.1)$ \\
\hline Average, ascending 1 and descending 1 & $15.6(7.5)$ & $21.0(10.7)$ & $17.1(9.1)$ \\
\hline \multicolumn{4}{|l|}{ Left leg } \\
\hline Ascending 1 & $15.9(8.0)$ & $21.3(10.9)$ & $17.2(9.3)$ \\
\hline Descending 1 & $14.7(7.8)$ & $19.5(10.8)$ & $15.9(9.0)$ \\
\hline Ascending 2 & $15.2(7.8)$ & $20.3(10.8)$ & $16.5(9.2)$ \\
\hline Average, all three & $15.3(7.7)$ & $20.3(10.6)$ & $16.5(9.0)$ \\
\hline Average, ascending & $15.6(7.8)$ & $20.8(10.6)$ & $16.9(9.0)$ \\
\hline Average, ascending 1 and descending 1 & $15.3(7.8)$ & $20.3(10.7)$ & $16.5(9.0)$ \\
\hline \multicolumn{4}{|l|}{ Average, left and right legs } \\
\hline Ascending 1 & $16.0(7.3)$ & $21.4(9.9)$ & $17.5(8.6)$ \\
\hline Descending 1 & $14.9(7.2)$ & $19.7(10.0)$ & $16.2(8.6)$ \\
\hline Ascending 2 & $15.2(7.2)$ & $20.5(10.0)$ & $16.6(8.8)$ \\
\hline All six measurements & $15.3(7.1)$ & $20.5(9.8)$ & $16.7(8.4)$ \\
\hline
\end{tabular}

Values are mean (SD)

of about 60 years with this difference between the sexes increasing with age.

Upper limb results Table 2 shows the mean (SD) of the distal arm measurements. There were 604 participants with a distal arm measurement. These values are reported separately for the left and right limbs, by age group. For both limbs separately, there were statistically significant differences in the total means between: (1) the first ascending measurement and the descending measurement $(p<0.001)$; (2) the first ascending measurement and the second ascending measurement $(p<0.001)$; and (3) the descending measurement and the second ascending measurement $(p<0.001)$. For both limbs, the mean of the first descending measurement was the highest value, followed by the second ascending measurement, then the descending measurement. In comparing the total means between the left and right limbs, there were statistically significant differences between the first ascending measurements $(p<0.001)$, the descending measurements $(p<0.001)$ and the second ascending measurements $(p<0.001)$. In all three comparisons between the left and right limbs, the right limb had the highest mean values. Note that only one participant had a metacarpal measurement and no participants had wrist measurements;
Fig. 1 Bland-Altman plot comparing the difference in measurements (first minus second ascending measurements, right toe) against the average in measurements (all three measurements, right toe). Continuous line, mean; dashed lines, $95 \%$ CIs

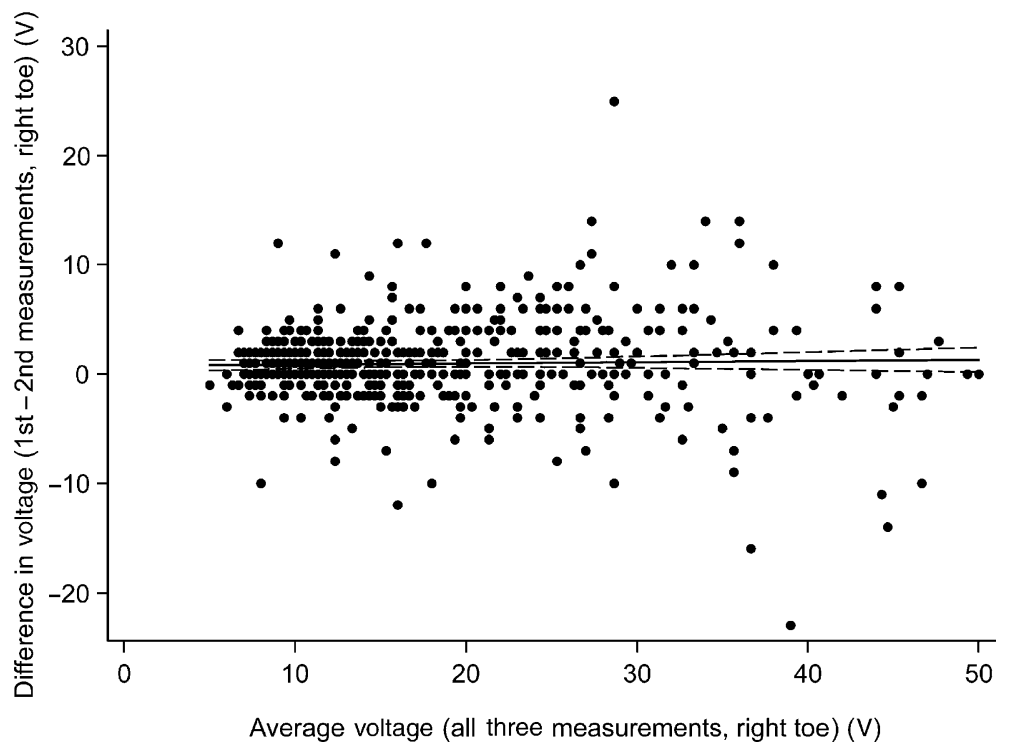


Fig. 2 Scatterplot of distal measurement (toes) against age (years), with indicators for sex (women, crosses; men, circles) and corresponding linear regression lines (women, continuous lines; men, dashed lines; including 95\% CIs). Linear regression equations: women, voltage $=-1.328+(0.242 \times$ age $)$; men, voltage $=-19.764+$ $(0.605 \times$ age $)$

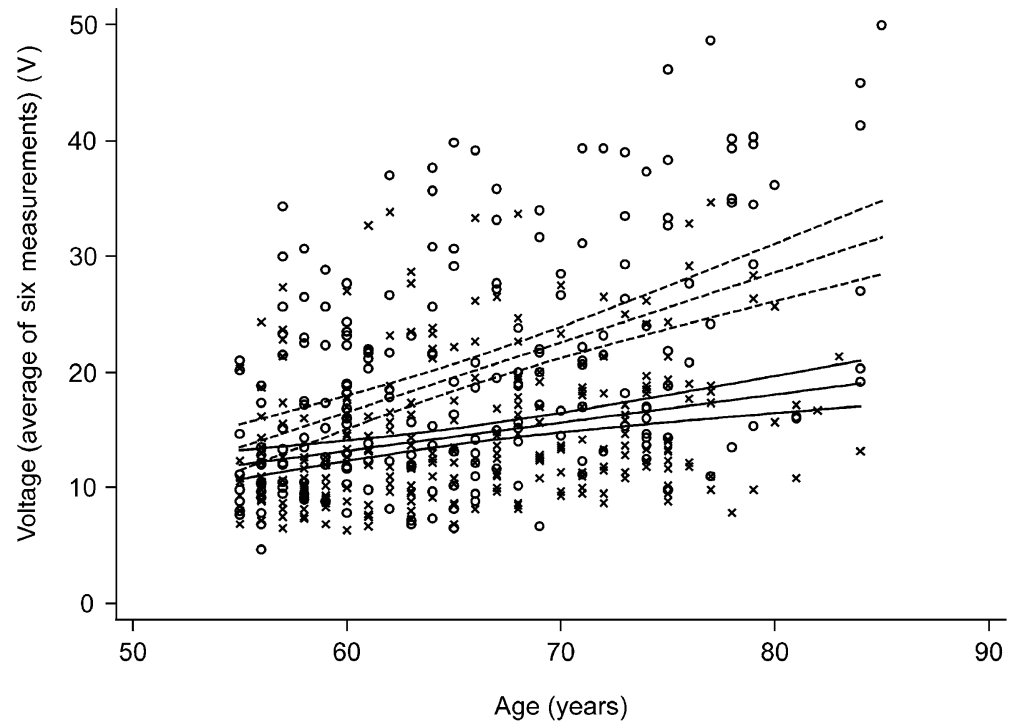

the metacarpal measurement was not analysed. A BlandAltman plot confirmed these findings (data not shown).

The relationship between the distal arm measurements and age, separately for both sexes, is shown in Fig. 3. There was a statistically significant linear relationship between distal arm measurements and age, for men $(p<0.001)$ and women $(p<0.001)$, with voltage increasing with age. However, there was no statistically significant difference in voltage between men and women.
Variability between left and right limb measurements There was a very high correlation between left and right limb distal measurements for finger $(r=0.79)$ and toe $(r=0.76)$, with only a few outliers. Bland-Altman plots of the difference between right and left readings for the lower limb indicate that the values for the right side tend to be higher than for the left side; this trend increases as the average voltage increases, as does the spread of the values (Fig. 4). This pattern was similar for the upper limb (data not shown).

Table 2 Distal measurements summary for left and right arm, stratified by participants aged $\leq 70$ years and those aged $>70$ years

\begin{tabular}{|c|c|c|c|}
\hline Distal measurement & $\leq 70$ years & $>70$ years & Total \\
\hline \multicolumn{4}{|l|}{ Right arm } \\
\hline Ascending 1 & $8.6(2.4)$ & $10.3(4.2)$ & $9.1(3.4)$ \\
\hline Descending 1 & $7.7(2.4)$ & $9.2(4.2)$ & $8.1(3.2)$ \\
\hline Ascending 2 & $7.9(2.3)$ & $9.6(4.8)$ & $8.4(3.5)$ \\
\hline Average, all three & $8.0(2.2)$ & $9.7(4.3)$ & $8.6(3.2)$ \\
\hline Average, ascending & $8.2(2.2)$ & $10.0(4.4)$ & $8.8(3.3)$ \\
\hline Average, ascending 1 and descending 1 & $8.1(2.3)$ & $9.7(4.1)$ & $8.6(3.2)$ \\
\hline \multicolumn{4}{|l|}{ Left arm } \\
\hline Ascending 1 & $8.1(2.1)$ & $9.8(4.1)$ & $8.7(3.4)$ \\
\hline Descending 1 & $7.3(2.2)$ & $8.6(3.9)$ & $7.8(3.3)$ \\
\hline Ascending 2 & $7.6(2.1)$ & $9.3(4.6)$ & $8.2(3.6)$ \\
\hline Average, all three & $7.7(2.0)$ & $9.2(4.0)$ & $8.2(3.3)$ \\
\hline Average, ascending & $7.8(2.0)$ & $9.5(4.2)$ & $8.5(3.4)$ \\
\hline Average, ascending 1 and descending 1 & $7.7(2.1)$ & $9.2(3.9)$ & $8.3(3.2)$ \\
\hline \multicolumn{4}{|l|}{ Average, left and right arms } \\
\hline Ascending 1 & $8.4(2.1)$ & $9.9(3.6)$ & $8.9(3.1)$ \\
\hline Descending 1 & $7.5(2.1)$ & $8.8(3.5)$ & $7.9(2.9)$ \\
\hline Ascending 2 & $7.7(2.0)$ & $9.4(4.3)$ & $8.3(3.3)$ \\
\hline All six measurements & $7.9(2.0)$ & $9.4(3.7)$ & $8.4(3.0)$ \\
\hline
\end{tabular}

Values are mean (SD) 
Fig. 3 Scatterplot of distal measurement (fingers) against age (years), with indicators for sex (women: crosses; men: circles) and corresponding linear regression lines (women: continuous lines; men: dashed lines; including $95 \% \mathrm{CIs}$ ). Linear regression equations: women, voltage $=$ $2.037+(0.092 \times$ age $)$; men, voltage $=2.810+(0.086 \times$ age $)$

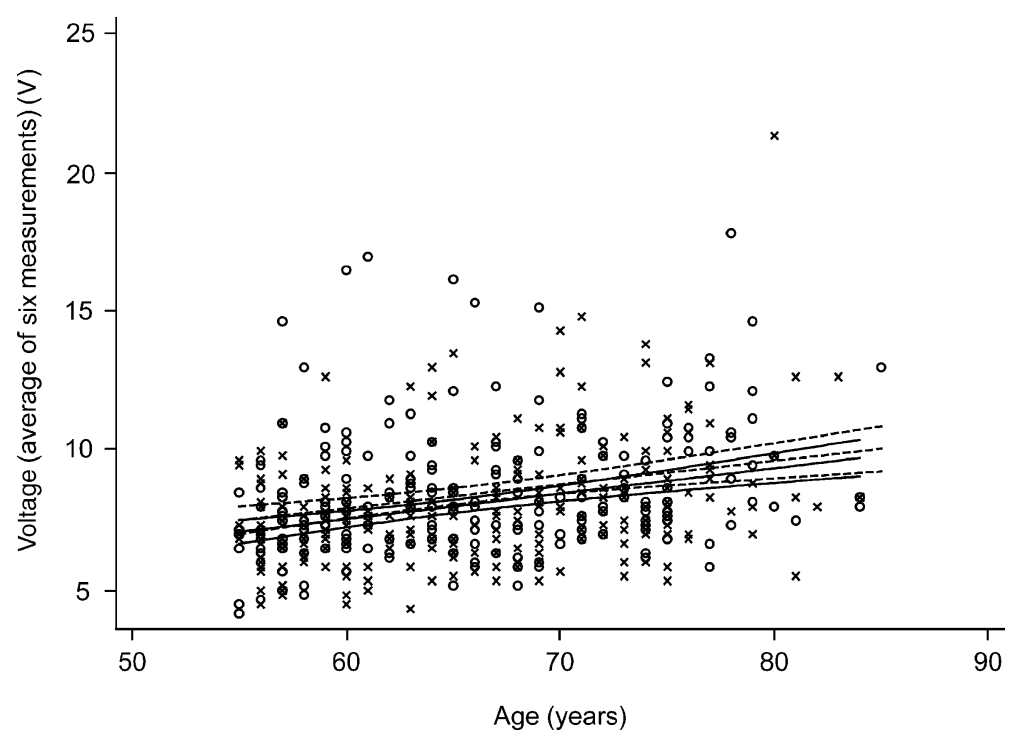

Previous investigations of variability have arrived at varying conclusions. Some studies found good intraobserver reliability, but poorer inter-observer reliability [33], with coefficients of variation up to $45 \%$ [34]. Other studies found good reproducibility [35] and good validity between centres [36]; this probably relates to good standardisation and adherence to testing protocols.

In our study, statistically significant differences were observed across the means for each reading with the highest reading recorded for the first ascending, followed by the second ascending and finally the descending reading. This was consistent for both lower and upper limb site testing. It is not unusual for the descending vibration perception reading to be lower than the ascending readings. The VPT recorded for the descending test is generally less distinct and the participant may occasionally find it difficult to determine when the vibration sensation has in fact disappeared [37]. Pacinian corpuscles have been identified as the receptors responsible for the transduction of vibratory energy [38], with increasing pressure of the vibrating stimuli resulting in an increase of the frequency discharge from Pacinian corpuscles [38]. Pacinian corpuscles can be susceptible to an adaptation effect whereby increasing vibration pressure results in increased radiation of the vibratory stimuli and excitation of the deeper receptors. To counter this effect, a rest period of at least 1 min between the second and third readings was adopted in the protocol. Other studies have found that a criterion shift can also occur, resulting in lower subsequent readings related perhaps to a learning response to the test [10]. VPT measurements were consistently higher for the right limbs and this might reflect the fact that the study protocol initiated the vibration measures on the right foot and right hand, respectively. 
Fig. 4 Bland-Altman plot comparing the difference in measurements (right minus left toe, first ascending measurements) against the average in measurements (all three measurements, right toe). Continuous line, mean; dashed lines, $95 \% \mathrm{CIs}$

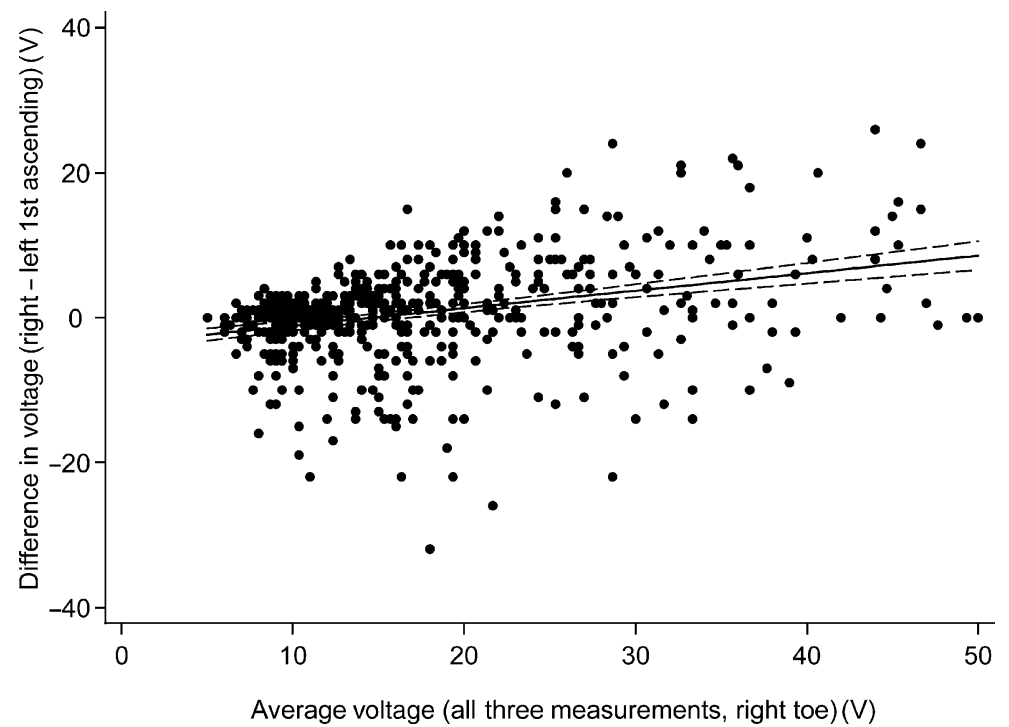

Previously published literature documents the effect of age $[10,37]$ and height $[10,28]$ on VPT measurements. For men and women we found statistically significant linear associations between the distal leg and distal arm VPT measurements (average of six readings across limbs) and age, with VPT increasing with age. No such association was found for height in this study (results not presented). Interestingly, from approximately 60 years of age, a statistically significant difference in VPT measures for the lower limb was observed between men and women, with men having significantly higher readings. While VPT measures increased linearly with age for distal arm sites, no statistically significant difference between men and women was observed with increasing age. Our results are consistent with previous studies that show ageing to be associated with a decline in vibration perception, with deterioration less marked in the hand than in the foot $[35,39]$.

When comparing adults of $\leq 70$ years with adults $>70$ years, the mean VPTs of all three measurements for both the toe and the finger were consistently higher for the older group. The variances as depicted by the standard deviation score were also slightly higher. This finding is supported by Thomson et al., who suggested VPT to be unreliable in the elderly due to the high variability in scores observed [40].

Conclusions This study provided an opportunity to describe the vibration perception threshold experience of older community-dwelling adults. While we were unable to exclude from the analyses participants who may have experienced a work-related exposure linked to peripheral nerve impairment, we did exclude participants with diseases such as diabetes (based on self-report and medication history), stroke and heart disease, all of which are known to be associated with underlying peripheral neurological impairment. It is, however, possible that some participants with early, undiagnosed diabetes were included in the sample, although this number is probably small.

Our results indicate that in clinical and research use, it may be sufficient to get the average of two ascending measures separated by at least $1 \mathrm{~min}$ in just the right hand or right foot, since these are usually the sites with the higher threshold. Although identical reference ranges can be used between men and women for the upper limb, there are significant differences in the lower limbs. The major determinant of VPT is age, and we were able to establish age-specific norms for VPT testing for adults aged 55 to 85 years.

Duality of interest The authors declare that there is no duality of interest associated with this manuscript.

\section{References}

1. Filley CM, Kleinschmidt-DeMasters BK (2001) Toxic leukoencephalopathy. N Engl J Med 345:425-432

2. Nasterlack M, Dietz MC, Frank KH et al (1999) A multidisciplinary cross-sectional study on solvent-related health effects in painters compared with construction workers. Int Arch Occup Environ Health 72:205-214

3. Broadwell DK, Darcey DJ, Hudnell HK, Otto DA, Boyes WK (1995) Work-site clinical and neurobehavioral assessment of solventexposed microelectronics workers. Am J Ind Med 27:677-698

4. Martyn CN, Hughes RA (1997) Epidemiology of peripheral neuropathy. J Neurol Neurosurg Psychiatry 62:310-318

5. Hughes RA (2002) Systematic reviews of treatment for inflammatory demyelinating neuropathy. J Anat 200:331-339

6. Tapp RJ, Shaw JE, de Courten MP, Dunstan DW, Welborn TA, Zimmet PZ, AusDiab Study Group (2003) Foot complications in Type 2 diabetes: an Australian population-based study. Diabetic Med 20:105-113

7. Mayfield JA, Sugarman JR (2000) The use of the SemmesWeinstein monofilament and other threshold tests for preventing foot ulceration and amputation in persons with diabetes. J Fam Pract 49(11 Suppl):S17-S29 
8. Adler AI, Boyko EJ, Ahroni JH, Stensel V, Forsberg RC, Smith DG (1997) Risk factors for diabetic peripheral sensory neuropathy. Results of the Seattle prospective diabetic foot study. Diabetes Care 20:1162-1167

9. Moody L, Arezzo J, Otto D (1986) Screening occupational populations for asymptomatic or early peripheral neuropathy. J Occup Med 28:975-986

10. Gerr F, Hershman D, Letz R (1990) Vibrotactile threshold measurement for detecting neurotoxicity: reliability and determination of age- and height-standardized normative values. Arch Environ Health 45:148-154

11. Pham H, Armstrong DG, Harvey C, Harkless LB, Giurini JM, Veves A (2000) Screening techniques to identify people at high risk for diabetic foot ulceration: a prospective multicenter trial. Diabetes Care 23:606-611

12. Dillman DA (1978). Mail and telephone surveys: the total design method. Wiley, New York

13. Ware JE, Sherbourne CD (1992) The MOS 36-item short form health survey (SF-36): I. Conceptual framework and item selection. Med Care 30:473-483

14. Washburn RA, Smith KW, Jette AM et al (1993) The physical activity scale for the elderly (PASE): Development and evaluation. J Clin Epidemiol 46:153-162

15. Washburn RA, McAuley E, Katula J et al (1999) The physical activity scale for the elderly (PASE); Evidence for validity. J Clin Epidemiol 52:643-651

16. Kessler R, Mroczek D (1994) Final version of our non-specific psychological distress scale [memo dated 10 March 1994]. Survey Research Center of the Institute for Social Research, University of Michigan, Ann Arbor. Available at: http://www.abs.gov.au/AUS STATS/abs@.nsf/ProductsbyCatalogue/B9ADE45ED60E0A1C CA256D2D0000A288?OpenDocument, accessed 7 August 2007

17. Andrews G, Slade T (2001) Interpreting scores on the Kessler psychological distress scale (K10). Aust N Z J Public Health 25:494-497

18. Radloff LS (1977) The CES-D scale: a self-report depression scale for research in the general population. Appl Psychol Meas 1:385-401

19. Weissman MM, Sholomskas D, Pottenger M et al (1977) Assessing depressive symptoms in five psychiatric populations: a validation study. Am J Epidemiol 106:203-214

20. Crook TH, Feher EP, Larrabee GJ (1992) Assessment of memory complaint in age-associated memory impairment: the MAC-Q. Int Psychogeriatr 4:165-175

21. Koenig HG, Westlund R, George LK et al (1993) Abbreviating the Duke social support index for use in chronically ill elderly individuals. Psychosomatics 34:61-69

22. Goodger B, Byles J, Higginbotham N et al (1999) Assessment of a short scale to measure social support among older people. Aust N Z J Public Health 23:260-265

23. Richardson J, Atherton Day N, Peacock S, Iezzi A (2004) Measurement of the quality of life for economic evaluation and the assessment of quality of life (AQoL) Mark 2 Instrument. Aust Econ Rev 37:62-88
24. Australian Bureau of Statistics (ABS) (2001) Population census, 2001. http://www.abs.gov.au/census, accessed 21 May 2007

25. Australian Bureau of Statistics (ABS) (2005) Population by age and sex, 2005. http://www.abs.gov.au/census, accessed 21 May 2007

26. Davis EA, Jones TW, Walsh P, Byrne GC (1997) The use of biothesiometry to detect neuropathy in children and adolescents with IDDM. Diabetes Care 20:1448-1453

27. Shy ME, Frohman EM, So YT et al (2003) Quantitative sensory testing: report of the therapeutics and technology assessment subcommittee of the American Academy of Neurology. Neurology 60:898-904

28. Bartlett G, Stewart JD, Tamblyn R, Abrahamowicz M (1998) Normal distributions of thermal and vibration sensory thresholds. Muscle Nerve 21:367-374

29. Inami K, Chiba K, Toyama Y (2005) Determination of reference intervals for vibratory perception thresholds of the lower extremities in normal subjects. J Orthop Sci 10:291-297

30. Hwu CM, Chang HY, Chen JY, Wang SL, Ho LT, Pan WH (2002) Quantitative vibration perception thresholds in normal and diabetic Chinese: influence of age, height and body mass index. Neuroepidemiology 21:271-278

31. Aaserud O, Juntunen J, Matikainen E (1990) Vibration sensitivity thresholds: methodological considerations. Acta Neurol Scand $82: 277-283$

32. Halonen P (1986) Quantitative vibration perception thresholds in healthy subjects of working age. Eur J Appl Physiol Occup Physiol 54:647-655

33. Peters EW, Bienfait HM, de Visser M, de Haan RJ (2003) The reliability of assessment of vibration sense. Acta Neurol Scand 107:293-298

34. Gelber DA, Pfeifer MA, Broadstone VL et al (1995) Components of variance for vibratory and thermal threshold testing in normal and diabetic subjects. J Diabetes Complications 9:170-176

35. de Neeling JN, Beks PJ, Bertelsmann FW, Heine RJ, Bouter LM (1994) Sensory thresholds in older adults: reproducibility and reference values. Muscle Nerve 17:454-461

36. Lindsell CJ, Griffin MJ (2003) Normative vibrotactile thresholds measured at five European test centres. Int Arch Occup Environ Health 76:517-528

37. Goldberg JM, Lindblom U (1979) Standardised method of determining vibratory perception thresholds for diagnosis and screening in neurological investigation. J Neurol Neurosurg Psychiatry 42:793-803

38. Vedel JP, Roll JP (1982) Response to pressure and vibration of slowly adapting cutaneous mechanoreceptors in the human foot. Neurosci Lett 34:289-294

39. Wiles PG, Pearce SM, Rice PJ, Mitchell JM (1990) Reduced vibration perception in right hands of normal subjects: an acquired abnormality? Br J Ind Med 47:715-716

40. Thomson FJ, Masson EA, Boulton AJ (1992) Quantitative vibration perception testing in elderly people: an assessment of variability. Age Ageing 21:171-174 